\title{
Magnetogenesis at Cosmic Dawn
}

\author{
J-B. Durrive ${ }^{1,2}$ and M. Langer ${ }^{1,2}$ \\ ${ }^{1}$ Institut d'Astrophysique Spatiale, Bâtiment 121, Univ. Paris-Sud, UMR8617, Orsay, F-91405 \\ ${ }^{2}$ CNRS, Orsay, F-91405. email: jdurrive@ias.u-psud.fr
}

\begin{abstract}
We present a mechanism for generating cosmological magnetic fields during the Epoch of Reionization, based on the photoionization of intergalactic hydrogen. A general formula is presented, together with an example numerical application which yields magnetic field strengths between $10^{-23}$ to $10^{-19} \mathrm{G}$ on intersource scales. This mechanism, which operates all along Reionization around any ionizing source, participates to the premagnetization of the whole intergalactic medium. Also, the spatial configuration of these fields may help discriminate them from those produced by other mechanisms in future observations.
\end{abstract}

Keywords. Cosmology:theory, magnetic fields, large-scale structure of universe

Revisiting Langer et al. (2005), we present an analytical model of cosmological magnetogenesis based on plasma-radiation interactions which occur during the Epoch of Reionization. As the first luminous objects formed, they emitted ionizing radiation which photoionized the neutral intergalactic medium (IGM), thus generating currents and inducing magnetic fields. The reionization of the Universe was accomplished by Population III stars, first galaxies and first quasars. Each source formed a fully ionized area (Strömgren sphere) around itself but long mean free path photons (UV and X) escaped into the IGM. Those photons knocked out free a fraction of bound electrons and transferred to them their momentum, generating radial currents and induced magnetic fields. But magnetic fields generated by adjacent currents compensate each other, unless these currents have different intensities. This condition is actually satisfied thanks to inhomogeneities in the IG M. Therefore magnetic fields are generated where the IGM is inhomogeneous. More precisely, we show in detail in Durrive \& Langer (2014) that sources generate outside their Strömgren spheres the following magnetic field:

$$
\vec{B}(t, \vec{r})=\frac{1}{e x_{e}}\left(N_{1} \frac{\vec{\nabla} x_{e}}{x_{e}}+N_{2} \vec{\nabla} \int_{r_{s}}^{r} n_{H I} d r\right) \times \frac{\hat{r}}{4 \pi r^{2}} t
$$

where, for $i=1,2, N_{i}(t, \vec{r})=\int_{\nu_{0}}^{\infty} f_{\mathrm{mt}} \sigma_{\nu}^{i} L_{\nu} e^{-\tau_{\nu}} d \nu$. In this expression, the first term is local while the second is global. They correspond to the necessity that two adjacent currents have different intensities. This is satisfied either when the matter configuration differs in two adjacent volume elements, or when the intensity of the ionizing radiation incident on two adjacent volume elements differs. This is reflected in the equation above, where the local term corresponds to local inhomogeneities in the electron fraction, and the global term corresponds to the transverse variation of photon absorption along adjacent lines-of-sight. In addition, we recover naturally the geometric dilution of photons, the strength being proportional to $r^{-2}$. Note also that the strength is linearly growing with time, essentially because we assumed a constant luminosity, and the mechanism operates until the source dies. $N_{1}$ and $N_{2}$ characterize the impact that the source has at distance $\vec{r}$ at time $t$. Indeed, they contain the fraction of momentum transferred from photons to electrons $f_{\mathrm{mt}}$, the photoionization cross section $\sigma_{\nu}$, the spectrum of the source $L_{\nu}$ and the optical depth $\tau_{\nu}$. 

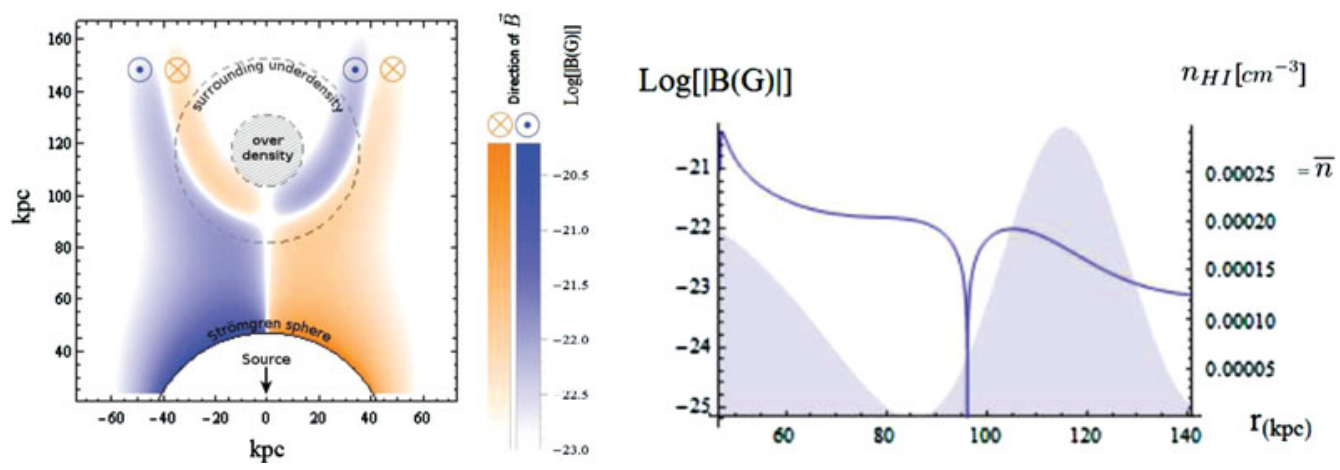

Figure 1. Left: Spatial distribution around an IGM inhomogeneity of the $B$-field (in Gauss) with a $z=10$ galaxy source. The symbols $\odot$ and $\otimes$ indicate that the field points towards and away from the reader, respectively. Right: Strength of the $B$-field along a path through the inhomogeneity (sketched in grey).

\begin{tabular}{|c|c|c|c|c|}
\hline Redshift & Source & $\log [B(G)]$ & scale $(k p c)$ & $\frac{1}{2} d_{\text {intersource }}(k p c)$ \\
\hline 30 & Pop III & $-19 /-21$ & $0.3 / 1$ & 10 \\
20 & & $-19 /-21$ & $0.5 / 1$ & \\
\hline 20 & First galaxy & $-20 /-22$ & $10 / 15$ & 25 \\
10 & & $-21 /-22$ & $30 / 100$ & \\
\hline 10 & Quasar & $-21 /-22$ & $300 / 1000$ & 1000 \\
6 & & $-22 /-23$ & $500 / 1500$ & \\
\hline
\end{tabular}

Figure 2. Typical values of the resulting fields and scales.

This analytical formula is valid for any ionizing source during Reionization. As an illustrative numerical application, we considered the case of Population III clusters and first galaxies with spectra computed with the Yggdrasil model (Zackrisson et al., 2011), as well as quasars with spectra fitted from observations (Shang et al., 2011), with a mildly non-linear inhomogeneity outside the Strömgren sphere of the source. The results are that, depending on their spectrum, luminosity, lifetime and epoch, different sources generate different magnetic fields (cf. figures 1 and 2). Namely, Pop III clusters generate stronger fields on short scales, while quasars magnetize less but over huge distances. First galaxies combine high amplitudes and large scales (intersource scales). Also, the geometrical configuration of the fields relfects the axisymmetry of the matter distribution with respect to the ionizing source, and fields generated in overdensities have opposite orientation to those generated in underdensities (cf. figure 1).

Conclusion. The mechanism presented here operates with any ionizing source, at any time during the Epoch of Reionization. The resulting magnetic strengths are comparable to those generated by other astrophysical mechanisms, but they appear on entire intersource distances. Therefore it contributes to the premagnetization of the whole Universe. Also, the specific spatial configuration of the generated fields might help discriminate from other cosmological magnetogenesis mechanisms with future observations (e.g. SKA) of the evolved magnetic fields.

\section{References}

Langer, M., Aghanim, N., \& Puget, J-L. 2005, A\& A, 443, 367-372

Durrive, J-B., \& Langer, M. 2014 (to be submitted)

Zackrisson, E., Rydberg, C.-E., Schaerer, D., Östlin, G., \& Tuli, M. 2003, ApJ, 740, 13

Shang, Z., Brotherton, M., Beverley, J., Wills, D. et al. 2011, ApJS, 196, 2 